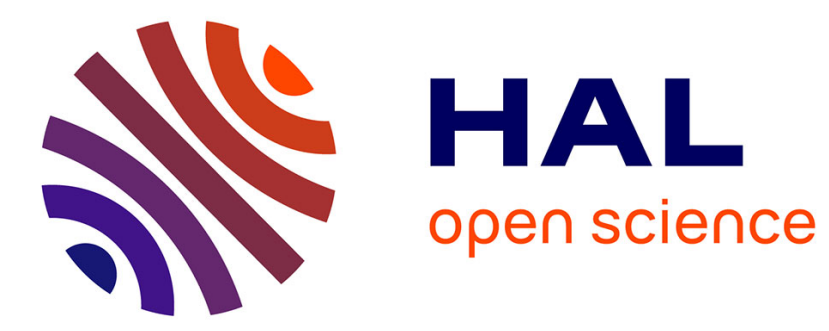

\title{
Modeling flow coating of colloidal dispersions in the evaporative regime: prediction of deposit thickness
}

\author{
Frédéric Doumenc, Jean Baptiste Salmon, Beatrice Guerrier
}

\section{To cite this version:}

Frédéric Doumenc, Jean Baptiste Salmon, Beatrice Guerrier. Modeling flow coating of colloidal dispersions in the evaporative regime: prediction of deposit thickness. Langmuir, 2016, 10.1021/acs.langmuir.6b02282 . hal-01416346

\section{HAL Id: hal-01416346 \\ https://hal.sorbonne-universite.fr/hal-01416346}

Submitted on 14 Dec 2016

HAL is a multi-disciplinary open access archive for the deposit and dissemination of scientific research documents, whether they are published or not. The documents may come from teaching and research institutions in France or abroad, or from public or private research centers.
L'archive ouverte pluridisciplinaire HAL, est destinée au dépôt et à la diffusion de documents scientifiques de niveau recherche, publiés ou non, émanant des établissements d'enseignement et de recherche français ou étrangers, des laboratoires publics ou privés. 


\title{
Modeling flow coating of colloidal dispersions in the evaporative regime: prediction of deposit thickness
}

\author{
Frédéric Doumenc, ${ }^{* \dagger, \ddagger}$ Jean-Baptiste Salmon, $₫$ and Béatrice Guerrier ${ }^{\dagger}$ \\ †Laboratoire FAST, Univ. Paris-Sud, CNRS, Université Paris-Saclay, F-91405, Orsay, \\ France \\ ‡Sorbonne Universités, UPMC Univ. Paris 06, UFR 919, 75005, Paris, France \\ ๆCNRS, Solvay, LOF, UMR 5258, Univ. Bordeaux, F-33600 Pessac, France \\ E-mail: doumenc@fast.u-psud.fr \\ Phone: +331691537 31. Fax: +33169158060
}

\section{Abstract}

We investigate flow coating processes, i.e., the formation of dry coatings starting from dilute complex fluids confined between a static blade and a moving substrate. In particular, we focus on the evaporative regime encountered at low substrate velocity, at which the coating flow is driven mainly by solvent evaporation in the liquid meniscus. In this regime, general arguments based on mass conservation show that the thickness of the dry film decreases as the substrate velocity increases, unlike the behavior in the well-known Landau-Levich regime. This work focuses on colloidal dispersions, which deserve special attention. Indeed, flow coating is expected to draw first a solvent-saturated film of densely packed colloids, which further dries fully when air invades the pores of the solid film. We first develop a model based on the transport equations for binary mixtures, which can describe this phenomenon continuously, using appropriate boundary conditions and a criterion to take into account pore-emptying in the colloidal film. Extensive numerical simulations of the model then demonstrate two regimes for the deposit thickness as a function of the process parameters (substrate velocity, evaporation rate, bulk concentration, and particle size). We finally derive an analytical model based on simplified transport equations that can reproduce the output of our numerical simulations very well. This model can predict analytically the two observed asymptotic regimes and therefore unifies the models recently reported in the literature.

\section{Introduction}

Making functional coatings starting from dilute colloidal dispersions or polymer solutions is a challenging issue for many industrial applications ranging from organic photovoltaic devices $^{1}$ to conductive films. ${ }^{2}$ Among the common techniques used to coat solid substrates, flow coating processes, such as blade coating, knife coating, or use of a doctor blade, have recently attracted considerable interest. Indeed, they generate possibilities not only for making functional coatings continuously, but also for patterning colloidal monolayers or ordered structures on a substrate, ${ }^{3-8}$ and even for generating films with thickness gradients. ${ }^{9}$

Figure 1a schematically depicts the specific case of blade coating: a film is drawn out of a liquid reservoir and confined by a nearly horizontal static blade and a substrate moving at a velocity $V$. A key issue concerns the prediction of the coating thickness $h_{d}$ as a function 

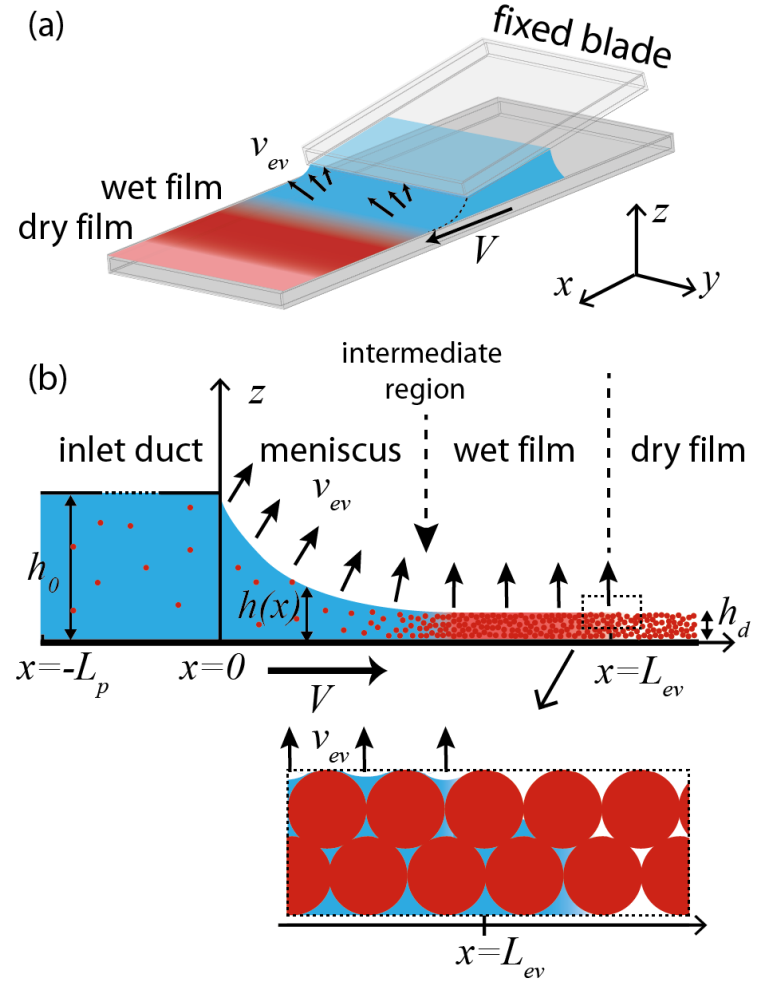

Figure 1: (a) Perspective and (b) side views of the flow coating behavior expected for a colloidal dispersion. A solvent-saturated film is drawn out of a liquid reservoir and maintained by capillary forces between a fixed blade and a substrate moving at velocity $V . v_{e v}$ is the local evaporation velocity. Pore-emptying occurs at $x=L_{e v}$, see also the zoom in the rectangle with dotted lines.

of the process parameters. Two main regimes were already identified: for large coating speeds $V$, a liquid film is drawn from the interplay between viscous and capillary forces (the so-called Landau-Levich regime), and the solvent later evaporates up to the formation of a solid coating. ${ }^{9}$ At low $V$, solvent evaporation within the liquid meniscus cannot be neglected, and solutes (or particles) contained in the liquid reservoir are concentrated up to the formation of a dry film. ${ }^{10,11}$ Quantitative modeling of this evaporative regime is particularly challenging, as it couples both hydrodynamics and physicochemical features of the investigated fluids.

Dimitrov and Nagayama ${ }^{10}$ provided a simple law based on mass conservation to account for the variations of the coating thickness $h_{d}$ with the process parameters,

$$
h_{d}=\frac{\varphi_{0}}{\varphi_{c}-\varphi_{0}} \frac{Q_{e v}}{V},
$$

where $\varphi_{c}$ is the solute volume fraction within the solid coating and $\varphi_{0}$ is the solute volume fraction within the liquid reservoir. $Q_{e v}$, the evaporation flux per unit of width, is defined as the integral of the local evaporation velocity $v_{e v}(\mathrm{~m} / \mathrm{s})$ over the evaporation region of length $L_{e v}$, i.e., $Q_{e v}=\int_{0}^{L_{e v}} v_{e v} d x\left(v_{e v} \neq 0\right.$ for $\left.0<x<L_{e v}\right)$. From eq 1 it follows that the proportionality relation $h_{d} \propto 1 / V$ is expected if $Q_{e v}$ does not depend on $V$. This proportionality relation has been actually reported recently by several groups for various systems, ${ }^{12-14}$ including polymer solutions, solutions of small molecules and colloidal dispersions. However it may fail for colloidal dispersions as the evaporation flux may depend on the substrate velocity $V$. Indeed, the coating flow is expected to first draw a wet film of densely packed colloids. Water flow remains significant in this porous packed bed, and evaporation vanishes only at a distance $L_{e v}$ from the blade. Evaporation stops (or strongly decreases) as the air/water menisci recede within the porous packed bed (see Figure 1). This mechanism occurs when the capillary forces cannot sustain the low solvent pressure imposed by evaporation-induced flow within the porous packed bed of colloids. ${ }^{15-17}$ As seen in the present study, $L_{e v}$ may depend significantly on the process parameters in some range of parameters. In flow coating, it may significantly impact prediction of the deposit thickness $h_{d}$ using eq 1 . Note that this region of highly concentrated solutes together with significant solvent flow does not exist in polymer solutions, since high concentrations of polymers induce strong decreases of both the mutual diffusion coefficient and the solvent chemical activity, unlike in colloidal dispersions. ${ }^{18}$

Recently, Jung and Ahn ${ }^{19}$ developed a model taking into account the wet film of densely packed colloids. However, their model neither accounts for previous experiments reporting the $h_{d} \propto 1 / V$ relation $^{13}$ nor succeeds in fitting accurately their own experimental results, as dis- 
cussed by the authors.

The results of the present work are twofold: (1) we first develop an original model based on the general transport equations for binary mixtures, which can describe continuously the formation of a dry coating starting from a dilute colloidal dispersion in a flow coating process. (2) We also provide more insights into the role played by the opening of the pores on the variation of the deposit thickness $h_{d}$ with the process parameters. More precisely, we derive a simple analytical prediction for $h_{d}$ taking into account this phenomenon, which is in very good agreement with our numerical simulations. Interestingly, this law exhibits two asymptotic behaviors: the first one is fully consistent with the $h_{d} \propto 1 / V$ relation already reported in some experiments, whereas the second one corresponds to the model proposed by Jung and Ahn. ${ }^{19}$

This work is organized as follows. In the next section, we detail our model based on transport equations using the lubrication approximation. We then discuss the crucial role played by boundary conditions, and we propose original conditions that enable continuous modeling of the colloidal consolidation in the flow coating process. We then solve this model numerically using a wide parametric study to capture the effect of the process control parameters on the deposit thickness $h_{d}$. In the last section, we thoroughly discuss the outputs of these numerical simulations, and we compare them to a simple analytical law based on conservation equations. The validity domain of this simplified model is specified, and previous experimental results ${ }^{13,19}$ are finally analyzed in light of this model.

\section{Model of evaporative flow coating of colloidal disper- sions}

\section{General description}

Our aim is to model flow coating of a colloidal dispersion on a substrate moving at a constant velocity $V$ in the evaporative regime (see Figure 1). A long inlet duct of length $L_{p}$ and constant height $h_{0}$ drives the dispersion from an infinite and perfectly stirred reservoir (located at $\left.x=-L_{p}\right)$ toward a free surface region $(x>0)$. The problem is assumed to be isothermal. Evaporation within the liquid meniscus, coupled to the flow induced by the moving substrate, concentrates the colloids up to the formation of a solvent-saturated densely packed array of colloids at a position $x_{f}$. This film is then simply transported by the moving substrate, as rearrangement of particles within the film is unlikely owing to the vanishing selfdiffusion of the colloidal particles at such high concentrations. ${ }^{20}$ This densely packed film later dries fully at a position $x \simeq L_{e v}$ when the solvent capillary forces can no longer sustain the receding of the air/solvent menisci within the porous packed bed.

\section{Evaporation velocity}

The evaporation velocity $v_{e v}(\mathrm{~m} / \mathrm{s})$ corresponds to the local volume flux of solvent, per unit of surface, owing to evaporation by diffusive drying normal to the air/dispersion surface. We assume, as is done by many authors, ${ }^{15,21,22}$ and experimentally evidenced in drying experiments (see for instance $\mathrm{ref}^{23}$ ), that pore emptying occurs before the concentration of colloids can significantly affect the saturated vapor pressure and thus the evaporation velocity $v_{e v}$ (at least for colloids with diameter $2 a>50 \mathrm{~nm}^{24}$ ). We also assume that evaporation vanishes suddenly for $x>L_{e v}$.

The diffusion in the gas phase may be $1 \mathrm{D}$ or $2 \mathrm{D}$, depending on the diffusive boundary layer thickness $\Lambda$ and the evaporation length $L_{e v}$. Indeed, the Laplace equation in the gas phase implies that 2D effects occur only at a distance on the order of $\Lambda$. Thus, for $L_{e v} \lesssim \Lambda$, a $2 \mathrm{D}$ model is required to describe the local solvent evaporation. An example of such a configuration is developed in ref ${ }^{18}$ for polymer solutions, and we will report in a future work a full model of evaporative flow coating of colloidal dispersions including these 2D effects. In the following, we consider for simplicity $\Lambda \ll L_{e v}$. Therefore, we assume that $v_{e v}$ is uniform over the entire surface of the film, except near $x=L_{e v}$ [more 
precisely, for $\left(L_{e v}-\Lambda\right) \lesssim x \leq L_{e v}$ ], where an increase in the evaporative flux is expected. ${ }^{25}$ However, the integral of the evaporative flux over this small distance scales as $\Lambda^{1 / 2}$, and we neglect this tip effect in the following.

To provide a continuous description of the expected behavior, we first provide the governing equations of our model within the liquid region, from the reservoir up to the opening of the pores. We then discuss the choice of appropriate boundary conditions, and finally we apply the model to the flow coating geometry.

\section{Governing equations}

\section{Mass balance}

We model a dispersion of monodisperse colloids of radius $a$ using the classical transport equations for a solvent/colloid binary mixture. ${ }^{26}$ The volume-averaged velocity of the mixture is defined as

$$
\mathbf{u}=\varphi \mathbf{u}_{\mathbf{p}}+(1-\varphi) \mathbf{u}_{\mathbf{s}}
$$

where $\mathbf{u}_{\mathbf{p}}$ and $\mathbf{u}_{\mathbf{s}}$ are the particle and solvent velocities, respectively, and $\varphi$ is the particle volume fraction. We assume that the partial specific volume of each component is constant, which leads to the local mass conservation $\nabla \cdot \mathbf{u}=0$.

The particle flux follows classically:

$$
\varphi \mathbf{u}_{\mathbf{p}}=\varphi \mathbf{u}-D(\varphi) \nabla \varphi
$$

where $D$ is the (long-time) collective diffusion coefficient of the mixture (defined here in the reference frame of the volume-averaged velocity). ${ }^{26}$ In eq 3 , we neglect shear-induced particle migration, which may also induce velocity drift between the particles and the solvent, particularly for large particles. ${ }^{27}$ The conservation equation for the particle concentration reads

$$
\partial_{t} \varphi+\mathbf{u} \nabla \varphi=\nabla \cdot(D(\varphi) \nabla \varphi) \text {. }
$$

In the description of the evaporative regime of blade coating of colloidal dispersions shown schematically in Figure 1, these conservation equations can be averaged in the liquid menis- cus over the liquid height $h$, leading to

$$
\begin{aligned}
\frac{\partial h}{\partial t}+\frac{\partial Q}{\partial x} & =-v_{e v}, \\
\frac{\partial(\varphi h)}{\partial t}+\frac{\partial Q_{p}}{\partial x} & =0,
\end{aligned}
$$

where $Q_{p}(x, t) \equiv \int_{0}^{h} \varphi u_{p} d z$ is the particle volume flux, $Q(x, t) \equiv \int_{0}^{h} u d z$ is the dispersion volume flux, and $v_{e v}$ is the evaporation velocity.

\section{Collective diffusion coefficient}

The collective diffusion coefficient in eq 3 results from an interplay between colloidal interactions within the solvent and hydrodynamic interactions at finite colloid concentrations. ${ }^{28}$ $D(\varphi)$ follows the generalized Stokes-Einstein relation:

$$
D(\varphi)=\varphi \frac{k}{\eta_{0}} \frac{\partial \Pi}{\partial \varphi}
$$

where $\eta_{0}$ is the solvent viscosity, $\Pi(\varphi)$ is the osmotic pressure, and $k(\varphi)$ is the permeability of the dispersion,

$$
\Pi(\varphi)=\frac{k_{B} T}{v_{p}} \varphi z(\varphi), \quad k(\varphi)=\frac{2 a^{2}}{9 \varphi} K(\varphi),
$$

where $k_{B}$ is Boltzmann's constant, $T$ is the temperature, $v_{p}=4 / 3 \pi a^{3}, z(\varphi)$ is the osmotic compressibility, and $K(\varphi)$ is the sedimentation factor. Therefore, relation 7 also reads

$$
D(\varphi)=D_{0} K(\varphi) \frac{\partial(\varphi z(\varphi))}{\partial \varphi}
$$

where $D_{0}=k_{B} T /\left(6 \pi \eta_{0} a\right)$ is the StokesEinstein diffusion coefficient.

In the following, we restrict our model to colloids interacting through a hard-sphere (HS) potential, ${ }^{29}$ and we use an analytical formula to model both $K(\varphi)$ and $z(\varphi)$. Concerning the sedimentation factor, we assume $K(\varphi)=$ $(1-\varphi)^{6}$ as in refs, ${ }^{20,29}$ and for the osmotic compressibility, we assume that

$$
z(\varphi)=\frac{1+a_{1} \varphi+a_{2} \varphi^{2}+a_{3} \varphi^{3}+a_{4} \varphi^{4}}{1-\varphi / \varphi_{c}},
$$

where $a_{1}=4-1 / \varphi_{c}, a_{2}=10-4 / \varphi_{c}, a_{3}=$ 
$18-10 / \varphi_{c}, a_{4}=\alpha / \varphi_{c}^{5}-18 / \varphi_{c}$, and $\alpha \simeq 1.5$, and $\varphi_{c} \simeq 0.64$ is the random close-packing volume fraction. This analytical formula ${ }^{20}$ was shown to asymptotically match the variations of $\Pi(\varphi)$ over the entire concentration range from the Carnahan-Starling regime at low concentration to the divergence of the osmotic compressibility approaching $\varphi_{c}$.

The divergence of the osmotic pressure and collective diffusion coefficient at the closepacked concentration $\varphi_{c}$ (which is related to the divergence of $z(\varphi)$ for a finite $K(\varphi)$ ) accounts for the formation of the wet film typical of colloidal dispersions. For simplicity, we consider an HS dispersion with a glass transition at $\varphi_{c}$. We thus do not include in our model any (reversible or not) transition from a sol to a solid gel phase (colloidal crystallization ${ }^{30}$ or densely coagulating ${ }^{21}$ or loosely flocculating gels $^{31}$ ) that may occur below the close packing. Indeed, transport within these solid phases can always be described by an effective diverging collective diffusion coefficient $D(\varphi)$, and the main features of our general results would not be strongly affected.

\section{Momentum balance and boundary condi- tions}

In several experimental cases (confined drops, ${ }^{32}$ unidirectional drying within capillaries or microchannels, ${ }^{21,33}$ flat evaporating films ${ }^{15,34}$ ), solvent evaporation occurs in a fixed geometry (or with a known evolution), with the assumption of 1D transfer. Therefore mass conservation $\nabla \cdot u=0$ entirely determines the velocity field $u$ and the transport equation eq (4) alone makes it possible to describe the concentration process.

When the collective diffusion coefficient $D(\varphi)$ diverges at a fixed concentration $\varphi_{c}$, the same equation leads to a continuous description of the liquid $\rightarrow$ solid transition (solid means here a densely packed wet film) as the divergence of $D(\varphi)$ prevents $\varphi$ overcoming $\varphi_{c}$ despite evaporation. In the above cited works, the knowledge of the mechanical equilibrium is not required to model the liquid $\rightarrow$ solid transition. However, $\nabla p=0$ is often implicitly assumed along with the concept of pervadic pressure defined as

$$
p_{l i q}=p-\Pi,
$$

where $p_{\text {liq }}$ corresponds to the pore pressure of the solvent within the dispersion. ${ }^{21}$ This approach allows the equivalence between the Darcy regime (within the solid) and Fick's law (within the liquid dispersion). ${ }^{20}$

An additional problem arises for flow coating processes, as well as drying of sessile drops and drying fronts in 2D evaporating films. Indeed the evaporating geometry is not known a priori and evolves during solvent evaporation. In that case, the flow profile $u$ and pressure $p$ have to be calculated using momentum balance equations (Stokes equations, $\nabla p \neq 0$ ) to predict the temporal evolution of the evaporating geometry. To the best of our knowledge, the liquid $\rightarrow$ solid transition has thus never been continuously modeled in such geometries due to this subtle coupling. To overcome this difficulty, the solutions of the above conservation equations coupled with the Stokes equations are often artificially truncated at a concentration $\varphi<\varphi_{c}$ in order to describe the entire drying process up to the solid phase. ${ }^{15,34}$ Style et al. have even taken a further step by including poro-elastic modeling to describe the drying of the colloidal solid under lateral constraints. ${ }^{21}$ More recently, Kaplan et al. also proposed a phenomenological relation between the particle and solvent volume fluxes to address these subtle issues. ${ }^{35,36}$ However, their resulting model does not respect the conservation equations for binary mixtures, and in particular global mass conservation.

We show in the following that specific boundary conditions for the standard momentum balance equations make it possible to model continuously both the concentration field and flow pattern within the liquid meniscus (dilute suspension) up to the formation of a solventsaturated and dense film in the flow coating experiment depicted in Figure 1. Note that in the wet film region shown in Figure 1, the free surface macroscopic curvature is negligible and $\nabla p=0$ (see below), leading to $\nabla \Pi=-\nabla p_{l i q}$. Our description of transport within the dense 
film using the Fick's law (see eqs 3 and 7) is thus strictly equivalent to the Darcy's description as one can indeed re-write eq 3 as

$$
(1-\varphi)\left(u_{s}-u_{p}\right)=-\frac{k}{\eta_{0}} \nabla p_{l i q}
$$

see for instance ref $^{37}$ for a full discussion.

Now we derive the momemtum balance equations for the free surface region (meniscus and wet film, $0<x<L_{e v}$ ). Disregarding inertia and gravity, the long-wave approximation leads to the following momentum balance:

$$
\begin{aligned}
& \frac{\partial p}{\partial x}=\eta(\varphi) \frac{\partial^{2} u}{\partial z^{2}} \\
& \frac{\partial p}{\partial z}=0
\end{aligned}
$$

where $z$ is the spatial coordinate normal to the substrate, $p$ is the pressure of the dispersion, and $\eta$ is its dynamic viscosity. In the above equations, we assume that $\varphi$ is homogeneous over the liquid height and that no additional terms (such as those proposed in refs ${ }^{38,39}$ involving the relative flow between the solvent and the particles) are needed to describe the entire range of concentrations, from the fluid phase to the solvent-saturated film.

We chose to model $\eta(\varphi)$ for our HS colloidal dispersion by the Krieger-Dougherty law:

$$
\eta=\eta_{0}\left(1-\varphi / \varphi_{c}\right)^{-\nu}
$$

where $\nu=2.5 \varphi_{c} \simeq 1.6 .^{40}$ The pressure of the dispersion (considered as a whole) is related to the curvature of the liquid film through Laplace's law: ${ }^{41}$

$$
p-p_{a t m}=-\gamma \frac{\partial^{2} h}{\partial x^{2}},
$$

where $\gamma$ is the air/dispersion surface tension and $\partial^{2} h / \partial x^{2}$ is the free surface macroscopic curvature, not to be confused with the microscopic curvature of pore menisci represented in Figure1b.

The integration of eqs 13 and 14 over the liquid height $h$ requires two boundary conditions, one at the solid/liquid interface, $z=0$, and the other at the liquid/gas interface, $z=h$.
Writing appropriate boundary conditions for a colloidal dispersion described as a continuous binary mixture is a subtle procedure. ${ }^{38}$ Indeed, the volume-averaged velocity $u$ and particle velocity $u_{p}$ (see eq 2 ) are defined properly only at a mesoscopic scale that makes the averaging of these quantities over many different colloidal particles possible. ${ }^{38}$

Assuming zero shear stress at the free surface, i.e., $\partial u / \partial z=0$ at $z=h$, and the classical noslip condition for the volume-averaged velocity $u=V$ at $z=0$ leads to the standard relation

$$
Q(x, t)=V h+\frac{h^{3} \gamma}{3 \eta} \frac{\partial^{3} h}{\partial x^{3}},
$$

where $\gamma$ is assumed constant (no Marangoni effect).

This last relation, in combination with mass conservation (eq 5), involves the following condition for a film deposited on a substrate in the steady regime $\left(\varphi \rightarrow \varphi_{c}\right)$ :

$$
V \frac{\partial h}{\partial x}=-v_{e v}
$$

as $\eta(\varphi) \rightarrow \infty$ and assuming that the pressure gradient does not diverge (we are looking for a regular solution). Such boundary conditions thus cannot describe the formation of a close-packed film with a fixed height $h$, as expected for such a densely packed bed of colloids with an infinite viscosity. Indeed, as the self diffusion coefficient of colloids approaches zero within a dense solvent-saturated film, ${ }^{20}$ we expect $u_{p}=V$ and $\varphi \simeq \varphi_{c}$, leading thus to $Q_{p}(x) \simeq \varphi_{c} V h(x)$. Solute conservation eq 6 , assuming the steady state, therefore implies a constant height $h$, which is not consistent with eq 18 above (in the evaporative regime, the order of magnitude of the ratio $v_{e v} / V$ can be as high as 1 in some cases).

If we now assume that the no-slip boundary condition at the moving substrate holds for the particle velocity only, i.e., $u_{p}=V$ at $z=0$, integration of eqs 13 and 14 now yields

$$
Q_{p}(x, t)=\varphi V h+\varphi \frac{h^{3} \gamma}{3 \eta} \frac{\partial^{3} h}{\partial x^{3}},
$$

and solute conservation (eq 6) within the dense 
film leads to

$$
V \frac{\partial h}{\partial x}=0
$$

i.e., the possibility of a close-packed film with a constant height $h$, as required.

Assuming this last set of boundary conditions, integration of eqs 13 and 14 coupled to the conservation eqs 5 and 6 leads to the following governing equations:

$$
\begin{aligned}
& \frac{\partial h}{\partial t}+\frac{\partial}{\partial x}\left(\frac{h^{3} \gamma}{3 \eta} \frac{\partial^{3} h}{\partial x^{3}}+\frac{h D}{\varphi} \frac{\partial \varphi}{\partial x}\right)+V \frac{\partial h}{\partial x}=-v_{e v} \\
& \frac{\partial(\varphi h)}{\partial t}+\frac{\partial}{\partial x}\left(\frac{\varphi h^{3} \gamma}{3 \eta} \frac{\partial^{3} h}{\partial x^{3}}\right)+V \frac{\partial(\varphi h)}{\partial x}=0 .
\end{aligned}
$$

These two relations differ from the usual description of a liquid film coupled to evaporation (as, for instance, in refs ${ }^{18,42,43}$ ) because of the specific boundary condition imposed at the liquid/solid substrate interface.

However, the difference between these two boundary conditions is important only for $\varphi \rightarrow$ $\varphi_{c}$ for a colloidal dispersion. Indeed, the characteristic Péclet number follows $\mathrm{Pe}=V L / D_{0} \gg$ 1 for $\varphi \ll \varphi_{c}$, where $L$ is a macroscopic length scale on the order of the meniscus size in our case, and $D_{0}$ is the diffusion coefficient in the dilute domain. Thus, we obtain $u \simeq u_{p}$ from eq 3 , and this result is confirmed by the output of our simulations (in particular Figure 5). These two boundary conditions nevertheless lead to two distinct descriptions in the concentrated regime, as the collective diffusion coefficient $D(\varphi)$ diverges for $\varphi \rightarrow \varphi_{c}$, leading to the possibility of $u \neq u_{p}$. Indeed, one expects that $u \neq u_{p}$ in the close-packed film advected by the moving substrate (i.e., $u_{p} \simeq V$ ), as evaporation possibly drives a relative solvent flow through the dense colloidal assembly, resulting in $u>V$.

\section{Liquid pressure and opening of the pores}

In the present approach, the liquid pressure $p_{l i q}$ is used as a criterion to characterize the transition from a solvent-saturated film to a dry film. Air/solvent menisci invade the colloidal wet film at a position $x=L_{e v}$, where the pore pressure $p_{l i q}$ (see eq 11) reaches a critical capillary pressure, see Figure1b. This condition reads

$$
p_{l i q}-p_{a t m}=-\kappa \gamma_{a} / a \quad \text { at } x=L_{e v},
$$

where $p_{a t m}$ is the atmospheric pressure, $\gamma_{a}$ is the air/water surface tension (which is assumed to be equal to $\gamma$, the air/dispersion surface tension), and $\kappa$ is a constant depending on the geometry of the colloidal packed bed. For monodisperse spheres, we use $\kappa=3 \varphi_{c} /(1-$ $\left.\varphi_{c}\right) \simeq 5.33$, as suggested in ref. ${ }^{31}$ Evaporation is assumed to be negligible for $x>L_{e v}$, as the invasion of the air/solvent menisci may rapidly desaturate the whole film thickness, or may add a possibly large resistance to diffusive mass transfer in the inert gas as suggested in refs. ${ }^{15,34}$

\section{Application to the flow coating ge- ometry}

In the following, we consider the flow coating geometry depicted in Figure 2 and restrict our analysis to the steady state. The domain of interest ranges from $x=-L_{p}$ to $x=L_{e v}$.

Two sets of governing equations must be considered: (i) those for the area within the inlet duct $\left(-L_{p}<x<0\right)$ and (ii) those for the free surface region $\left(0<x<L_{e v}\right)$. In the latter region, we use the governing equations 21 22 , which describe in a continuous way the colloidal suspension drying from the dilute regime (meniscus) to the concentrated regime (closepacked film). As shown later in the Numerical Results section, our model predicts a very sharp transition from the dilute dispersion to the dense film. In the following we note $x_{f}$ the position of the liquid $\rightarrow$ solid transition, with $x_{f}$ estimated from the abscissa of the maximum of the volume fraction spatial derivative.

Actually, the numerical resolution on the entire domain raises a difficulty, because the position of the boundary $L_{e v}$ is not known a priori but results from the determination of the liquid pressure field. To simplify the numerical resolution by avoiding the resolution of a moving boundary problem, the wet close-packed film is 


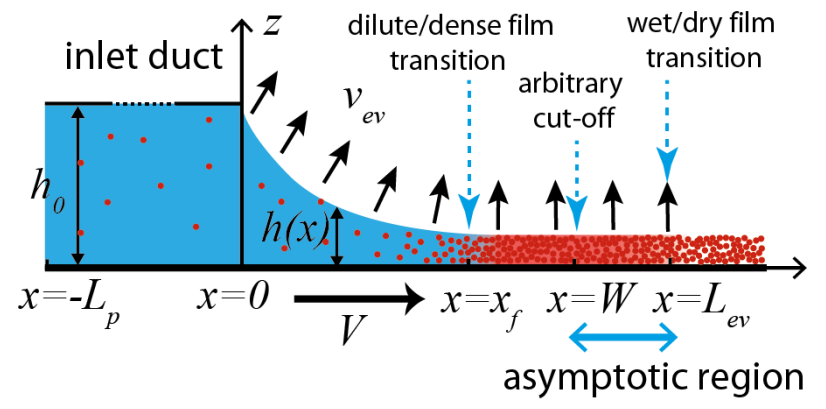

Figure 2: Position of the solid $\rightarrow$ liquid transition front $x_{f}$, lower limit of the asymptotic region $W$ and evaporation length $L_{e v}\left(x_{f}<W<\right.$ $\left.L_{e v}\right)$. Note that $x_{f}$ is an output of our numerical model.

cut at a fixed arbitrary length $W$ such that the particle volume fraction is assumed to be very close to $\varphi_{c}$ (but not strictly equal) in the range $W<x<L_{e v}$ (see Figure 2). This assumption allows us to derive an asymptotic version of governing equations 21-22, valid in the range $W<x<L_{e v}$, and simple enough to get (i) an analytical expression giving $L_{e v}$ as a function of $h(W)$ and $\varphi(W)$ (ii) boundary conditions at $x=W$. With these two information, the numerical resolution can be performed in the fixed-boundary-domain $-L_{p}<x<W$ instead of $-L_{p}<x<L_{e v}$.

The governing equations for these different domains, the matching conditions at their intersections $x=0$ and $x=W$, and the boundary conditions at $x=-L_{p}$ and $x=L_{e v}$ are provided in the following subsections. Then the asymptotic model is used to shift the boundary conditions from $x=L_{e v}$ to $x=W$.

Governing equations in the inlet duct $\left(-L_{p}<x<0\right)$

The governing equation for the concentration field in the inlet duct is obtained from eqs 2,5 , and 6 , assuming $h=h_{0}$ and $v_{e v}=0$ :

$$
\frac{Q_{0}}{h_{0}} \frac{\partial \varphi}{\partial x}=\frac{\partial}{\partial x}\left(D \frac{\partial \varphi}{\partial x}\right),
$$

where $Q_{0}$ is the liquid flux in the inlet duct, which is independent of $x$ because of eq 5 .

The suspension in the inlet duct being dilute, the pressure drop between the points $x=-L_{p}$ and $x=0$ is disregarded, i.e., $p(x=0) \simeq p(x=$ $\left.-L_{p}\right)=p_{0}$.

\section{Governing equations in the free surface} region $\left(0<x<L_{e v}\right)$

The governing equations in the free surface region are eqs 21-22 with $\partial_{t} h=0$. They are used without further simplification in the range $0<x<W$. Conversely, in the domain $W<x<L_{e v}$, we assume that the particle volume fraction field verifies

$$
\varphi(x) \simeq \varphi_{c}-\varepsilon(x) \text { with } \varepsilon(x) \rightarrow 0,
$$

which implies $\eta \rightarrow \infty$ (from eq 15), $D \rightarrow \infty$ (from eqs 9-10) and $\partial_{x} \varphi \simeq-\partial_{x} \varepsilon \rightarrow 0$. Introducing the expression 25 in governing equations 21-22 and keeping the leading order terms yields

$$
\begin{aligned}
& \frac{\partial}{\partial x}\left[h D(\varphi) \frac{\partial \varphi}{\partial x}\right]=-\varphi_{c} v_{e v} \\
& \frac{\partial h}{\partial x}=0
\end{aligned}
$$

Remarkably enough, although the particle volume fraction gradient goes to zero, the diffusion flux in eq 26 does not vanish because of the divergence of the diffusion coefficient. Note that with the assumption 25, the expression of the osmotic compressibility 10 simplifies to

$$
z(\varphi)=\frac{\alpha}{\varphi_{c}-\varphi}
$$

Injecting eq 28 in eq 9 along with eq 25 yields a simpler expression of the diffusion coefficient, to be used in the region such that $W<x<L_{e v}$ :

$$
D(\varphi)=D_{0} \frac{\alpha \varphi_{c}\left(1-\varphi_{c}\right)^{6}}{\left(\varphi_{c}-\varphi\right)^{2}}
$$

Another worth noticing consequence of assumption 25 is

$$
p \simeq p_{a t m} \quad \text { for } \quad W<x<L_{e v},
$$

because of eqs 16 and 27 (no macroscopic curvature in the flat close-packed film). 


\section{Matching conditions}

At the connection between the inlet duct and the meniscus $(x=0)$, we write the continuity of $Q, Q_{p}$, and $\varphi$. In addition, the liquid height verifies the conditions

$$
h=h_{0} \text { and } \frac{\partial^{2} h}{\partial x^{2}}=C_{0} \text { at } x=0,
$$

where the curvature $C_{0}$ is deduced from Laplace's law, $C_{0}=\left(p_{a t m}-p_{0}\right) / \gamma$.

At $x=W$, five matching conditions are provided by writing the continuity of the height $h$, slope $\partial h / \partial x$, and curvature $\partial^{2} h / \partial x^{2}$ on one hand, and the continuity of the solute volume fraction $\varphi$ and its spatial derivative $\partial \varphi / \partial x$ on the other hand. With the help of condition 25 (which is supposed to be valid at $x=W$ ), one can easily show that these continuity relations implicitly contain the global mass conservation $Q\left(W^{-}\right)=Q\left(W^{+}\right)$and particle mass conservation $Q_{p}\left(W^{-}\right)=Q_{p}\left(W^{+}\right)$.

\section{Boundary conditions}

The inlet duct is in contact with a reservoir at constant particle volume fraction $\varphi_{0}$, so

$$
\varphi=\varphi_{0} \text { at } x=-L_{p}
$$

We need two boundary conditions at $x=L_{e v}$ because $L_{e v}$ is unknown. Neglecting the water transfer in the almost-dried porous medium at $x>L_{e v}$ yields a first boundary condition,

$$
\frac{\partial \varphi}{\partial x}=0 \text { at } x=L_{e v}
$$

A second boundary condition arises from the pore opening condition 23. With the help of eqs 11 and 30, eq 23 can be rewriten $\Pi\left(\varphi_{d}\right) \simeq$ $\kappa \gamma / a$. Using the expression 28 for the dimensionless compressibility factor, we see that the pore opening transition occurs at a particle volume fraction $\varphi_{d}$ given by

$$
\varphi_{d}=\frac{\varphi_{c}}{1+3 \alpha k_{B} T /\left(4 \pi \kappa \gamma a^{2}\right)} .
$$

Numerical estimates show that $\varphi_{d} \simeq \varphi_{c}(1-\epsilon)$, where $\epsilon=3 \alpha k_{B} T /\left(4 \pi \kappa \gamma a^{2}\right) \ll 1$, which makes eq 34 consistent with the assumption 25 .

\section{Dimensionless equations}

The boundary conditions 31 suggest the choices of $h_{0}$ for the liquid height scale and $L \equiv$ $\sqrt{h_{0} / C_{0}}$ for the abscissa scale. Notice that the latter quantity is on the order of magnitude of the meniscus length $L_{m}$. Indeed, a parabola of curvature $C_{0}$ and height $h_{0}$ at $x=0$ intercepts the $x$ axis with zero slope at the abscissa, $x=\sqrt{2} L$. Using the time scale $\tau \equiv L^{2} / D_{0}$, we obtain $D_{0} / L$ for the scale of the liquid velocity component parallel to the substrate. Introducing these expressions into eqs 5 and 21 brings out the other scalings. The other dimensionless quantities are $\tilde{v}_{e v}=v_{e v} /\left(D_{0} h_{0} / L^{2}\right)$, $\tilde{Q}=Q /\left(D_{0} h_{0} / L\right), \tilde{V}=V /\left(D_{0} / L\right), \tilde{D}=D / D_{0}$, $\tilde{\eta}=\eta / \eta_{0}$, and $\tilde{L}_{m}=\sqrt{2}$. The dimensionless version of the governing equations is given in the following.

In the inlet duct $\left(-\tilde{L}_{p}<\tilde{x}<0\right)$, we obtain

$$
\tilde{Q}_{0} \frac{\partial \varphi}{\partial \tilde{x}}=\frac{\partial}{\partial \tilde{x}}\left(\tilde{D} \frac{\partial \varphi}{\partial \tilde{x}}\right) .
$$

The full equations for the free surface region $(0<\tilde{x}<\tilde{W})$ read

$$
\begin{aligned}
& \delta \frac{\partial}{\partial \tilde{x}}\left(\frac{\tilde{h}^{3}}{\tilde{\eta}} \frac{\partial^{3} \tilde{h}}{\partial \tilde{x}^{3}}\right)+\frac{\partial}{\partial \tilde{x}}\left(\frac{\tilde{h} \tilde{D}}{\varphi} \frac{\partial \varphi}{\partial \tilde{x}}\right)+\tilde{V} \frac{\partial \tilde{h}}{\partial \tilde{x}}=-\tilde{v}_{e v} \\
& \delta \frac{\partial}{\partial \tilde{x}}\left(\frac{\varphi \tilde{h}^{3}}{\tilde{\eta}} \frac{\partial^{3} \tilde{h}}{\partial \tilde{x}^{3}}\right)+\tilde{V} \frac{\partial(\varphi \tilde{h})}{\partial \tilde{x}}=0
\end{aligned}
$$

where

$$
\delta=\frac{\gamma h_{0}^{2} C_{0}}{3 \eta_{0} D_{0}} .
$$

In the asymptotic region $\left(\tilde{W}<\tilde{x}<\tilde{L}_{e v}\right)$, the equations reduce to

$$
\begin{aligned}
& \frac{\partial}{\partial \tilde{x}}\left[\tilde{h} \tilde{D} \frac{\partial \varphi}{\partial \tilde{x}}\right]=-\tilde{v}_{e v} \varphi_{c} \\
& \frac{\partial \tilde{h}}{\partial \tilde{x}}=0
\end{aligned}
$$

They are supplemented by the dimensionless counterpart of the matching conditions and 
boundary conditions.

\section{Shift of the boundary conditions from $\tilde{x}=\tilde{L}_{e v}$ to $\tilde{x}=\tilde{W}$}

Integration of eq 39 shows that the liquid height is constant in the close-packed region. Using height continuity at $\tilde{x}=\tilde{W}$, we obtain

$$
\tilde{h}(\tilde{x})=\tilde{h}\left(\tilde{W}^{-}\right) .
$$

Integrating relation 38 once, using the boundary condition $\partial \varphi / \partial \tilde{x}=0$ at $\tilde{x}=\tilde{L}_{e v}$, and using eq 40 leads to

$$
\frac{d \varphi}{d \tilde{x}}=\frac{\tilde{v}_{e v} \varphi_{c}}{\tilde{D}(\varphi) \tilde{h}\left(\tilde{W}^{-}\right)}\left(\tilde{L}_{e v}-\tilde{x}\right) .
$$

A second integration using the dimensionless expression of eq 29 and the continuity of the volume fraction at $\tilde{x}=\tilde{W}$ yields

$$
\begin{aligned}
& \frac{\varphi(\tilde{x})-\varphi\left(\tilde{W}^{-}\right)}{\left(\varphi_{c}-\varphi(\tilde{x})\right)\left(\varphi_{c}-\varphi\left(\tilde{W}^{-}\right)\right)}= \\
& \frac{\tilde{v}_{e v}\left[\tilde{L}_{e v}(\tilde{x}-\tilde{W})-\left(\tilde{x}^{2}-\tilde{W}^{2}\right) / 2\right]}{\alpha\left(1-\varphi_{c}\right)^{6} \tilde{h}\left(\tilde{W}^{-}\right)} .
\end{aligned}
$$

Using $\varphi=\varphi_{d}$ at $\tilde{x}=\tilde{L}_{e v}$ in eq 42 and solving the resulting algebraic equation gives the expression

$$
\tilde{L}_{e v}=\tilde{W}+\sqrt{2 \Phi H}
$$

where

$$
\begin{aligned}
\Phi & =\frac{\varphi_{d}-\varphi\left(\tilde{W}^{-}\right)}{\left(\varphi_{c}-\varphi_{d}\right)\left(\varphi_{c}-\varphi\left(\tilde{W}^{-}\right)\right)}, \\
H & =\frac{\tilde{h}\left(\tilde{W}^{-}\right) \alpha\left(1-\varphi_{c}\right)^{6}}{\tilde{v}_{e v}} .
\end{aligned}
$$

The concentration gradient at $\tilde{x}=\tilde{W}$ is deduced from relations 41, 29, and 43-45:

$$
\frac{d \varphi}{d \tilde{x}}=\left(\varphi_{c}-\varphi\left(\tilde{W}^{-}\right)\right)^{2} \sqrt{\frac{2 \Phi}{H}} \text { at } \tilde{x}=\tilde{W} .
$$

The other two boundary conditions are provided by the continuity of the slope and cur- vature at $\tilde{x}=\tilde{W}$ along with eq 40 :

$$
\frac{\partial \tilde{h}}{\partial \tilde{x}}=0 \quad \text { and } \quad \frac{\partial^{2} \tilde{h}}{\partial \tilde{x}^{2}}=0 \text { at } \tilde{x}=\tilde{W} .
$$

\section{Numerical resolution}

The governing equations for the domain $-\tilde{L}_{p}<$ $\tilde{x}<\tilde{W}$ are solved using Comsol, a commercial finite element software package (Galerkin method, quadratic Lagrange elements). Practically, the domain $\tilde{W}<\tilde{x}<\tilde{L}_{e v}$ is excluded from the simulation. One chooses a priori the length $\tilde{W}$ and imposes at $\tilde{x}=\tilde{W}$ the three boundary conditions $46-47$, which implicitly contain eqs 38-39, the matching conditions at $x=\tilde{W}$, and the boundary conditions at $x=\tilde{L}_{e v}$. Once the simulation has been completed, eqs 40 and 42 are used to get $\tilde{h}(\tilde{x})$ and $\varphi(\tilde{x})$ in the asymptotic region, i.e. the region such that $\tilde{W}<\tilde{x}<$ $\tilde{L}_{e v}$ which has been excluded from the simulation. Eq 43 provides $\tilde{L}_{e v}$.

A requirement for $\tilde{L}_{e v}$ to exist is $(\Phi H)>0$, which necessitates $\varphi_{d}>\varphi(\tilde{W})$. This condition is satisfied if $\tilde{W}<\tilde{L}_{e v}$. On the other hand, $\tilde{W}$ must be in the close-packed film. To ensure these two requirements, one starts a first simulation with a small value of $\tilde{W}$. Then a new simulation is performed with a larger value of $\tilde{W}$, and so on until the results become insensitive to $\tilde{W}$, which ensures that $\tilde{W}$ is located in the close-packed film. The rapid convergence of the procedure is illustrated in Figure 3, which shows that the results do not depend on $\tilde{W}$ as soon as $\tilde{W}$ is slightly larger than $\sqrt{2}$ (the meniscus size).

\section{Numerical results and discus- sion}

\section{Local description: liquid height, concentration, and velocity profiles}

We chose water as the solvent, with properties taken from ref $^{44}$ at temperature $T=298.15 \mathrm{~K}$, $\eta_{0}=0.89 \mathrm{mPa} . \mathrm{s}$, and $\gamma=72 \mathrm{mN} . \mathrm{m}^{-1}$. The geometrical characteristics are $h_{0}=100 \mu \mathrm{m}$, $C_{0}=2000 \mathrm{~m}^{-1}$, and $L_{p}=1 \mathrm{~cm}$, which results 


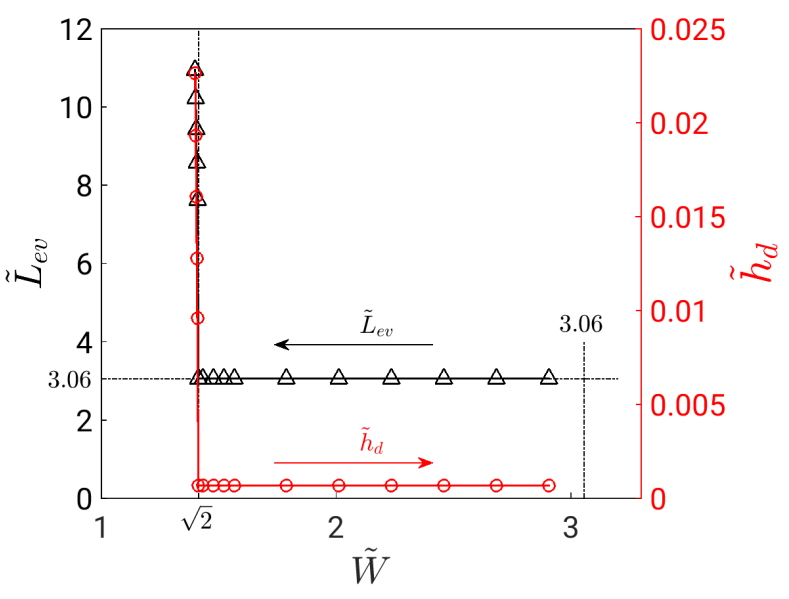

Figure 3: Effect of the arbitrary length $\tilde{W}$ on the calculated evaporation length $\tilde{L}_{e v}$ and deposit thickness $\tilde{h}_{d}$. Data set $\# 1$ with $\tilde{V}=$ $1.37 \times 10^{3}$ (see Table 1$)$.

in $L=223.6 \mu \mathrm{m}$ and $\tilde{L}_{p}=44.7$. These numerical values are kept constant throughout the paper. A parametric study was performed by varying four parameters: the substrate velocity $V$, evaporation velocity $v_{e v}$, particle diameter $2 a$, and bulk particle volume fraction $\varphi_{0}$. The data sets used in the numerical simulations are presented in Table 1.

Figure 4 displays some profiles obtained using data set $\# 9$. The results show three successive regions. The first one, $0<\tilde{x} \lesssim \sqrt{2}$, corresponds to a quasi-constant-curvature meniscus with weak variation of the solute concentration. It is followed by a thin transition zone characterized by a very strong concentration gradient, which coincides with a strong curvature peak (not represented on the figures). The last region is the flat water-saturated film with a concentration close to $\varphi_{c}$. Although the particle volume fraction field $\varphi(\tilde{x})$ is a continuous function of $\tilde{x}$, the transition from the dilute regime to the dense film is sharp. As previously said, the position of the liquid $\rightarrow$ solid transition $\tilde{x}_{f}$ is defined as the abscissa of the $\partial_{\tilde{x}} \varphi$ maximum.

The dispersion and particle velocities averaged over the thickness $[\tilde{U}(\tilde{x})=\tilde{Q} / \tilde{h}$ for the dispersion and $\tilde{U}_{p}(\tilde{x})=\tilde{Q}_{p} /(\tilde{h} \varphi)$ for the particles] are compared in Figure 5. The velocities are very similar in the meniscus region (large Péclet number, see eq 3) and then diverge in the transition region. Indeed, the mean particle velocity is equal to the substrate velocity $\tilde{V}$ in the close-packed film, where evaporation still induces significant solvent transport.
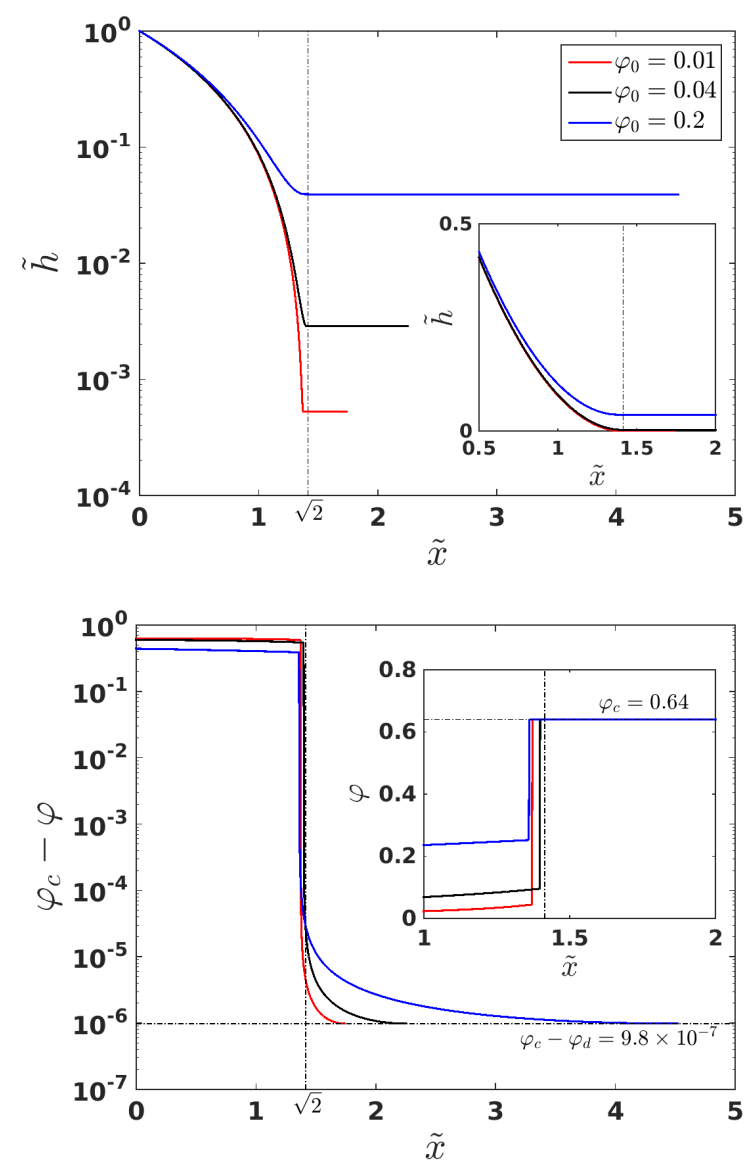

Figure 4: Liquid height profile $\tilde{h}(\tilde{x})$ (top) and particle concentration profile $\varphi_{c}-\varphi(\tilde{x})$ (bottom) for three values of the bulk particle volume fraction $\varphi_{0}$. Inset: magnified view of the intermediate region for $\varphi(\tilde{x})$. Data set \#9 (Table 1).

\section{Parametric study}

Figures 6-9 show the effect of the control parameters $\left(\tilde{V}, \tilde{v}_{e v}, a\right.$, and $\left.\varphi_{0}\right)$ on the dry film thickness $\tilde{h}_{d}=\tilde{h}\left(\tilde{x}=\tilde{L}_{e v}\right)$, evaporation length $\tilde{L}_{e v}$, and front position $\tilde{x}_{f}$. This latter quantity corresponds to the maximum of the spatial derivative of the solute concentration. For each data set, only one of the control parameters was varied, as detailed in Table 1 . The results are 
Table 1: Data sets used in the simulations. Four control parameters are considered. Only one dimensional parameter (indicated in bold characters) varies in each data set.

\begin{tabular}{|c|c|c|c|c|c|c|c|}
\hline Set $\#$ & $V / \mu \mathrm{m} . \mathrm{s}^{-1}$ & $\tilde{V}$ & $v_{e v} / \mu \mathrm{m} . \mathrm{s}^{-1}$ & $\tilde{v}_{e v}$ & $2 a / \mathrm{nm}$ & $\left(\varphi_{c}-\varphi_{d}\right) \times 10^{7}$ & $\varphi_{0} \times 100$ \\
\hline 1 & {$[\mathbf{1 , 8 0}]$} & {$\left[\mathbf{4 6 , 3 . 6} \times \mathbf{1 0}^{\mathbf{3}}\right]$} & 0.0162 & 1.65 & 100 & 9.83 & 10 \\
\hline 2 & {$[\mathbf{0 . 1 2}, \mathbf{6 5}]$} & {$\left[\mathbf{5 . 5 , 2 . 9} \times \mathbf{1 0}^{\mathbf{3}}\right]$} & 0.0162 & 1.65 & 100 & 9.83 & 1 \\
\hline 3 & {$[\mathbf{0 . 5}, \mathbf{1 9 4}]$} & {$\left[\mathbf{2 3 , 8 . 8} \times \mathbf{1 0}^{\mathbf{3}}\right]$} & 0.256 & 26.1 & 100 & 9.83 & 1 \\
\hline 4 & 1 & 45.6 & {$\left[\mathbf{1 . 6} \times \mathbf{1 0}^{-4}, \mathbf{1 . 6}\right]$} & {$[\mathbf{0 . 0 1 6 5}, \mathbf{1 6 5}]$} & 100 & 9.83 & 1 \\
\hline 5 & 30 & $1.37 \times 10^{3}$ & {$\left[\mathbf{6 . 4} \times \mathbf{1 0}^{-\mathbf{3}}, \mathbf{6 5}\right]$} & {$[\mathbf{0 . 6 6 , 6 . 6} \times \mathbf{1 0}$} & 100 & 9.83 & 1 \\
\hline 6 & 1 & {$[4.56,182]$} & 0.256 & {$[2.61,104]$} & {$[\mathbf{1 0}, \mathbf{4 0 0}]$} & {$[\mathbf{0 . 6 1 4}, \mathbf{9 8 3}]$} & 1 \\
\hline 7 & 30 & {$\left[137,5.47 \times 10^{3}\right]$} & 0.256 & {$[2.61,78.2]$} & {$[\mathbf{1 0}, \mathbf{3 0 0}]$} & {$[\mathbf{1 . 0 9}, \mathbf{9 8 3}]$} & 1 \\
\hline 8 & 1 & 45.6 & 0.256 & 26.1 & 100 & 9.83 & {$[\mathbf{0 . 0 5}, \mathbf{3 . 5}]$} \\
\hline 9 & 30 & $1.37 \times 10^{3}$ & 0.256 & 26.1 & 100 & 9.83 & {$[\mathbf{0 . 5}, \mathbf{4 0}]$} \\
\hline
\end{tabular}

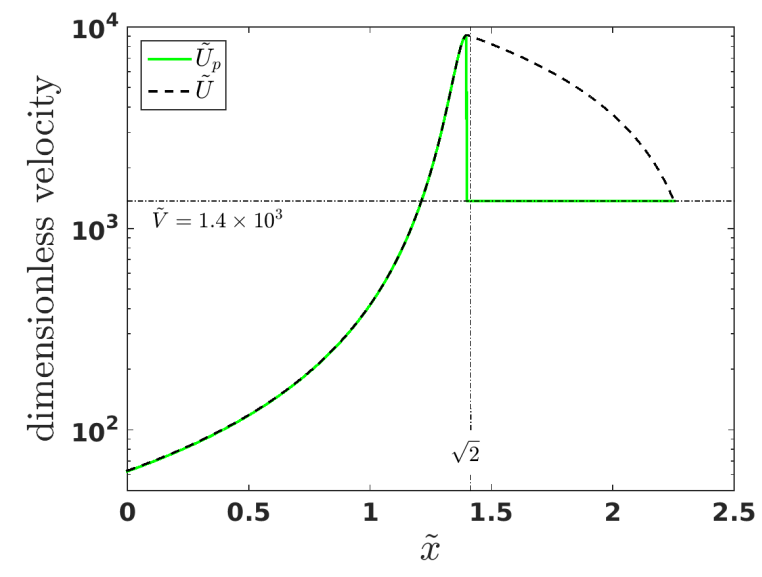

Figure 5: Instance of the averaged-thicknessvelocity profiles $\tilde{U}(\tilde{x})=\tilde{Q} / \tilde{h}$ (mean velocity) and $\tilde{U}_{p}(\tilde{x})=\tilde{Q}_{p} /(\tilde{h} \varphi)$ (particle velocity). Data set $\# 9$ (Table 1) with $\varphi_{0}=0.04$.

compared with the relation proposed by Dimitrov and Nagayama ${ }^{10}$ for the thickness of the dry film. According to their model, it should be proportional to the evaporation rate and particle concentration, and inversely proportional to the substrate velocity, but it should not depend on the particle size.

\section{Influence of substrate velocity (Figure 6)}

For the configuration studied in this work, i.e. a $1 \mathrm{D}$ model in the gas phase, it can be seen that the evaporative length increases significantly as the substrate velocity decreases. Consequently, the evaporation flux $\tilde{Q}_{e v}$ depends on $\tilde{V}$; therefore, the usual proportionality relation $\tilde{h}_{d} \propto 1 / \tilde{V}$ no longer holds at low velocities. This discrepancy is more pronounced at high $\varphi_{0}$ (Set $\left.\# 1\right)$. The thickness dependance rela-
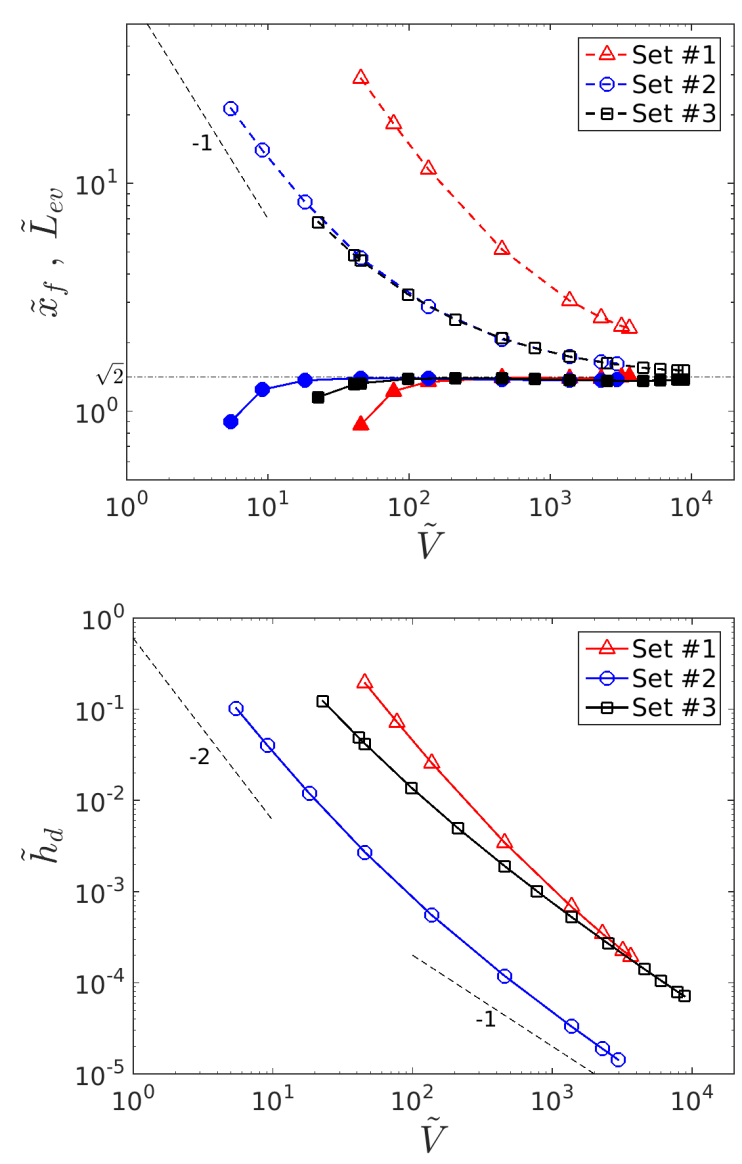

Figure 6: Effect of the substrate velocity $\tilde{V}$. Top: Concentration front position $\tilde{x}_{f}$ (solid symbols) and evaporation length $\tilde{L}_{e v}$ (open symbols) Bottom: Deposit thickness $\tilde{h}_{d}$. 
tion seems to shift from $\propto 1 / \tilde{V}$ to $\propto 1 / \tilde{V}^{2}$ as the substrate velocity decreases. It will be confirmed in the next section dedicated to the derivation of an analytical model.
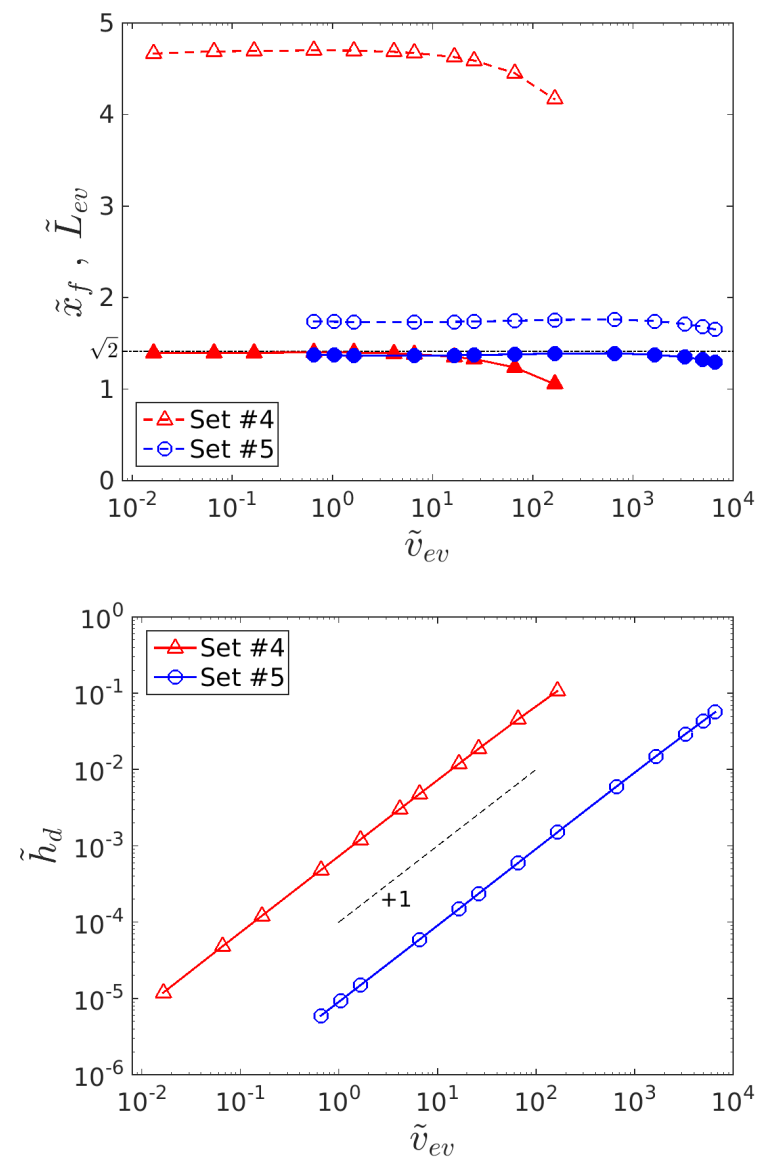

Figure 7: Effect of the evaporation velocity $\tilde{v}_{e v}$. Top: Concentration front position $\tilde{x}_{f}$ (solid symbols) and evaporation length $\tilde{L}_{e v}$ (open symbols). Bottom: Deposit thickness $\tilde{h}_{d}$.

\section{Influence of evaporation velocity (Figure} 7)

Numerical simulations show that $\tilde{L}_{e v}$ is quasiconstant in the range of $\tilde{v}_{e v}$ explored, so the proportionality relation $\tilde{h}_{d} \propto \tilde{v}_{e v}$ holds for the entire parameter range.

\section{Influence of particle size (Figure 8)}

According to the relation of Dimitrov and $\mathrm{Na}$ gayama, the thickness should not depend on the particle size. The results for set \#7 are in agreement with this scaling, whereas it is clearly not
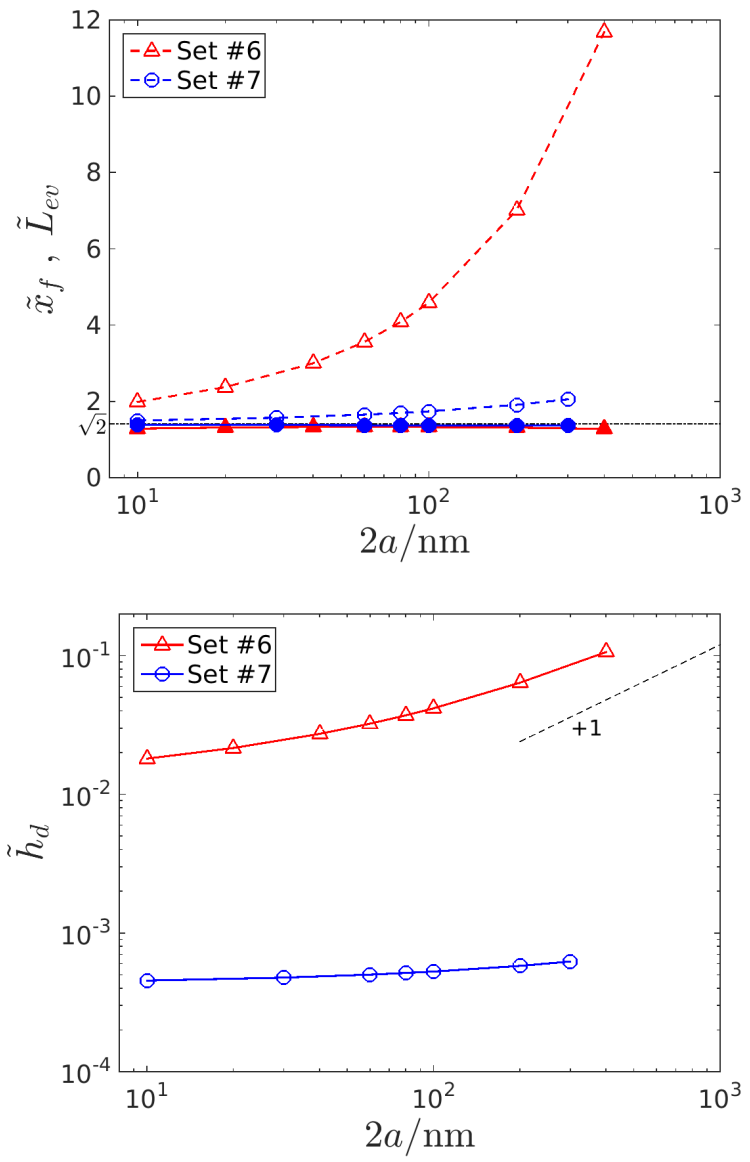

Figure 8: Effect of the particle diameter $a$. Top: Concentration front position $\tilde{x}_{f}$ (solid symbols) and evaporation length $\tilde{L}_{e v}$ (open symbols). Bottom: Deposit thickness $\tilde{h}_{d}$. 
satisfied for set $\# 6$. Note that the effect of the particle size is not trivial, as changing the radius of the particles induces changes in the diffusion coefficient $D(\varphi)$ and osmotic pressure $\Pi(\varphi)$ on the one hand, and in the criterion characterizing the opening of the pores (eq 23) on the other hand. No significant effect on the evaporation length is observed for data set $\# 7$. For data set $\# 6$ (low velocity and large particles), one may infer that the large permeability in that case lowers the pressure drop sufficiently so that the water-saturated film can extend to a greater length, despite the change in the criterion for pore emptying.
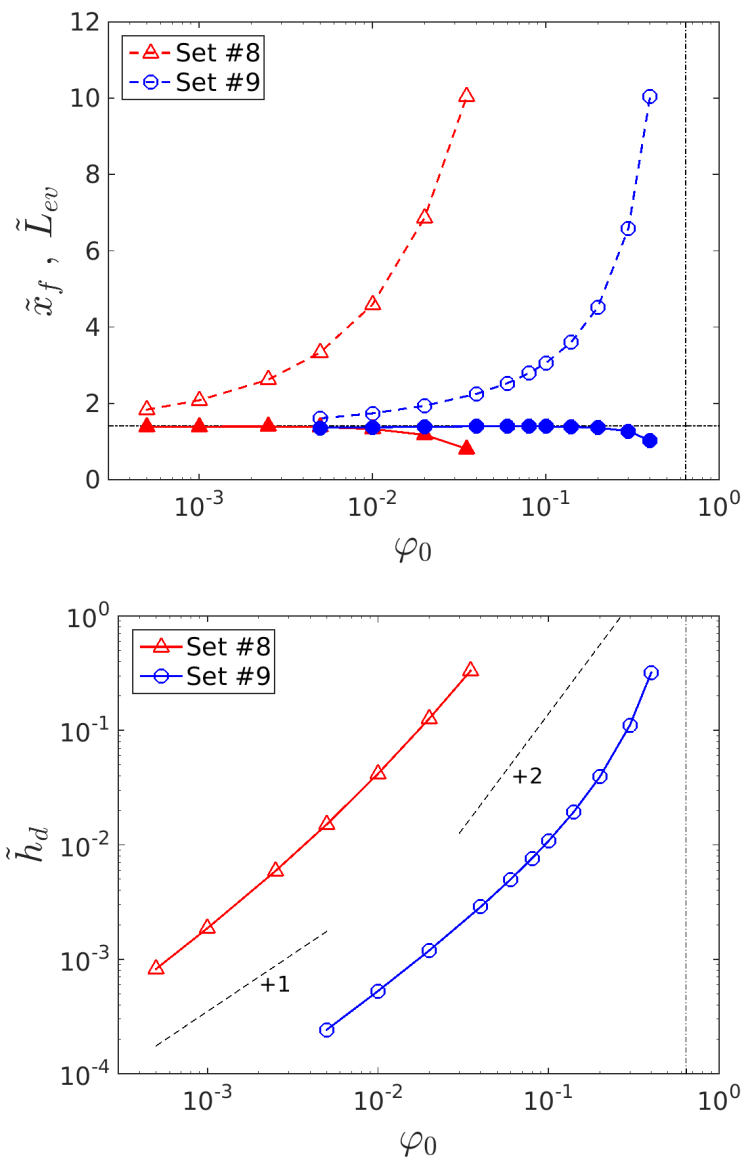

Figure 9: Effect of the bulk particle volume fraction $\varphi_{0}$. Top: Concentration front position $\tilde{x}_{f}$ (solid symbols) and evaporation length $\tilde{L}_{e v}$ (open symbols). Bottom: Deposit thickness $\tilde{h}_{d}$.

\section{Influence of particle concentration (Fig-} ure 9)

The last control parameter considered is the bulk particle volume fraction $\varphi_{0}$. Here again, a correlation between $\varphi_{0}$ and $\tilde{L}_{e v}$ is observed, which modifies the usual proportionality relation $\tilde{h}_{d} \propto \varphi_{0}$. This effect is expected, as an increase in $\varphi_{0}$ induces a thicker film, which facilitates transport of water in the dense closepacked film.

Note that in all the performed simulations, the position of the front $\tilde{x}_{f}$ shows very little sensitivity to the control parameters. This result will be used in the simplified model developed in the next section. Indeed, the extension of the evaporative length is not correlated with a strong displacement of the front position $\tilde{x}_{f}$, which always remains close to the meniscus length, i.e., $\tilde{x}_{f} \simeq \sqrt{2}$. It is only for a large deposit thickness, typically for $\tilde{h}_{d}=h_{d} / h_{0} \gtrsim 0.1$, that the front position retreats. One can infer that when the film thickness becomes of the same order as the inlet duct, there is no more meniscus, and thus $\tilde{x}_{f}$ is no longer constrained.

\section{Simple analytical model for pre- dicting the deposit thickness}

\section{Model derivation}

We now derive a simple analytical expression to predict the deposit thickness $\tilde{h}_{d}$ and evaporation length $\tilde{L}_{e v}$ in the steady-state regime. Following Dimitrov and Nagayama, ${ }^{10}$ a first relation between $\tilde{h}_{d}$ and $\tilde{L}_{e v}$ is obtained from a global mass balance over the fluid included in the entire domain from $\tilde{x}=-\tilde{L}_{p}$ to $\tilde{x}=\tilde{L}_{e v}$. The mass balance of the solvent/particle mixture reads

$$
\tilde{Q}\left(-\tilde{L}_{p}\right)=\tilde{L}_{e v} \tilde{v}_{e v}+\tilde{h}_{d} \tilde{V},
$$

which means that part of the fluid flux $\tilde{Q}\left(-\tilde{L}_{p}\right)$ entering the domain through the inlet duct evaporates, whereas the other part is removed by the substrate motion. As the particles do not evaporate, the particle mass balance reduces to

$$
\tilde{Q}_{p}\left(-\tilde{L}_{p}\right)=\varphi_{c} \tilde{h}_{d} \tilde{V}
$$


Assuming negligible diffusion at $\tilde{x}=-\tilde{L}_{p}$ yields $\tilde{Q}_{p}\left(-\tilde{L}_{p}\right)=\varphi_{0} \tilde{Q}\left(-\tilde{L}_{p}\right)$. From eqs 48 and 49 , we finally obtain

$$
\tilde{h}_{d}=\frac{\varphi_{0}}{\varphi_{c}-\varphi_{0}} \frac{\tilde{v}_{e v}}{\tilde{V}} \tilde{L}_{e v} .
$$

One recovers the standard Dimitrov and Nagayama law when $\tilde{L}_{e v} \simeq \tilde{L}_{m}=\sqrt{2}$. As shown above, $\tilde{L}_{e v}$ evolves with the process parameters for colloidal dispersions, and a second equation is thus required.

As discussed in the previous section, the concentration front is located around $\tilde{x}_{f} \simeq \sqrt{2}$. Thus, we set $\tilde{W} \simeq \tilde{L}_{m}=\sqrt{2}$ in eqs 43 45 relating the evaporation length $\tilde{L}_{e v}$. We then consider the assumptions $\tilde{h}(\sqrt{2}) \simeq \tilde{h}_{d}$ and $\varphi_{c}-\varphi\left(\tilde{L}_{e v}\right) \ll \varphi_{c}-\varphi(\sqrt{2})$, which are clearly supported by the numerical simulations (see Figure 4 , where the term $\varphi_{c}-\varphi(\tilde{x})$ decreases by 6 orders of magnitude from $\tilde{x}=\sqrt{2}$ to $\tilde{x}=\tilde{L}_{e v}$ ). Finally, we obtain

$$
\tilde{L}_{e v}=\sqrt{2}\left(1+\sqrt{\frac{\tilde{h}_{d} \alpha\left(1-\varphi_{c}\right)^{6}}{\tilde{v}_{e v}\left(\varphi_{c}-\varphi_{d}\right)}}\right) .
$$

This equation may also be found by considering the solvent flow relative to the colloids in the film. Indeed, one can show that eq 51 also reads (with real units)

$$
L_{e v}=L_{m}+L_{c}=L_{m}+\sqrt{\frac{2 h_{d} k\left(\varphi_{c}\right)}{\eta_{0} v_{e v}} \frac{\kappa \gamma}{a}}
$$

by using the definition of the permeability $k$ given in eq 8 and the approximation for $\varphi_{c}-\varphi_{d}$ given in eq 34 . The term $L_{c}$ in eq 52 corresponds to the position of the pore-emptying front in a drying colloidal film of thickness $h_{d}$ classically estimated from the balance between the viscous dissipation induced by solvent flow through the pores of the densely packed array of colloids (mass conservation, $h_{d} \partial_{x} v(x)=-v_{e v}$, and Darcy law, $\left.v(x)=-\left(k / \eta_{0}\right) \partial_{x} p_{\text {liq }}\right)$ and the capillary pressure $P_{c a p}=\kappa \gamma / a$; see, for instance, eq 13 in ref. ${ }^{16}$

Inserting eq 51 into eq 50 yields a second- degree algebraic equation whose solution is

$$
\tilde{h}_{d}=\mathcal{N}\left(1+\mathcal{M}+\sqrt{(1+\mathcal{M})^{2}-1}\right),
$$

where the following two dimensionless numbers have been introduced:

$$
\begin{aligned}
\mathcal{N} & =\frac{\varphi_{0}}{\left(\varphi_{c}-\varphi_{0}\right)} \frac{\tilde{v}_{e v}}{\tilde{V}} \sqrt{2} \\
\mathcal{M} & =\frac{\varphi_{0}}{\left(\varphi_{c}-\varphi_{0}\right) \tilde{V}} \frac{\alpha\left(1-\varphi_{c}\right)^{6}}{\left(\varphi_{c}-\varphi_{d}\right) \sqrt{2}}
\end{aligned}
$$

Finally, one obtains the expression for $\tilde{L}_{e v}$ by inserting eq 53 into eq 50:

$$
\tilde{L}_{e v}=\sqrt{2}\left(1+\mathcal{M}+\sqrt{(1+\mathcal{M})^{2}-1}\right) .
$$

We see in Figure 10 that eqs 53 and 56 make it possible to define master curves for the deposit thickness and evaporation length. The outputs of our numerical simulations collapse perfectly onto these master curves, validating the assumptions of our simplified model.

The limits of this simplified model must be recalled. It holds for the evaporative regime, i.e., for low substrate velocities, beyond the Landau-Levich regime. The study is restricted to a $1 \mathrm{D}$ regime in the gas phase, which means that the evaporation length was assumed to be larger than the diffusion boundary layer in the gas phase. Finally, the simplified model assumes a constant value for the position of the concentration front $x_{f} \simeq L_{m}$. According to the numerical simulations, this is valid for $h_{d} \lesssim h_{0} / 10$. This last condition may become untrue for very large bulk solute concentrations or very low substrate velocities.

\section{Asymptotic regimes}

Our detailed numerical simulations confirm that a simple picture emerges to describe the flow coating process of colloidal dispersions (see Figure 1): a liquid meniscus with a low colloid concentration is connected at a position $x_{f} \simeq L_{m}$ to a water-saturated film at $\varphi \simeq \varphi_{c}$. This film is then advected by the moving substrate, and the relative solvent flow through the pores of the densely packed colloids (induced by 

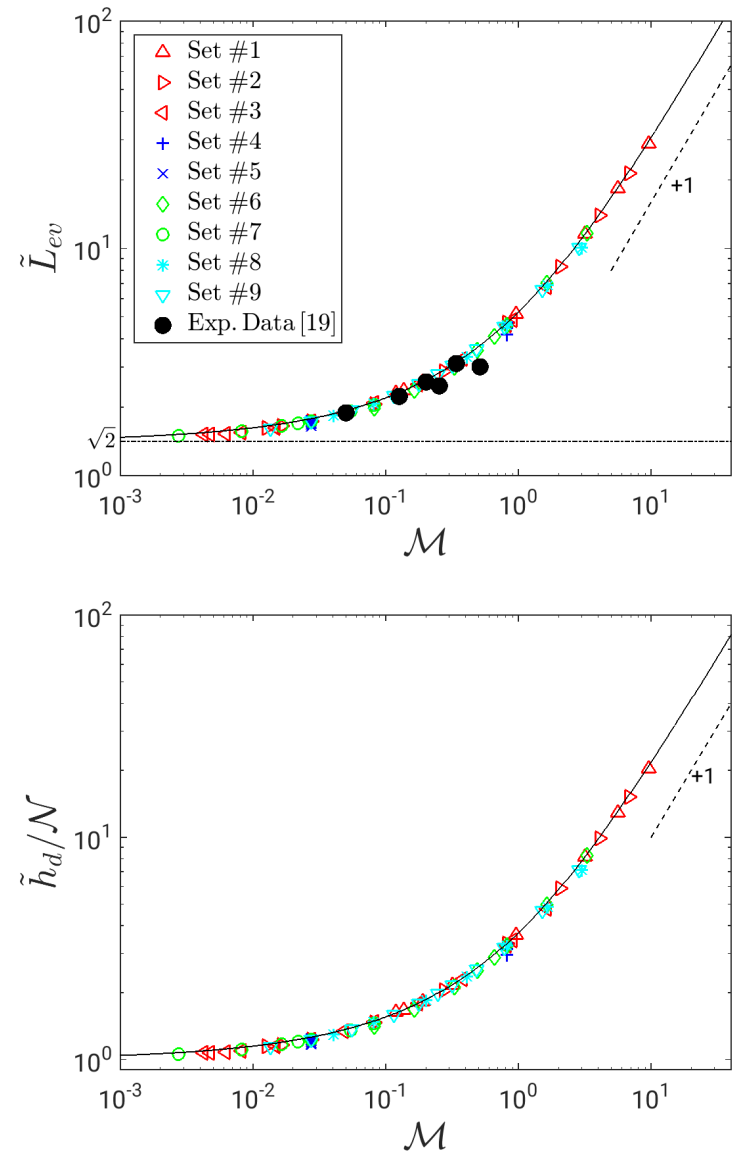

Figure 10: Evaporation lengths $\tilde{L}_{e v}$ and normalized deposit thicknesses $\tilde{h}_{d} / \mathcal{N}$ calculated from the numerical resolution of our model for the entire set of data displayed in Table 1. These data collapse perfectly onto the two master curves given by eqs 53 and 56 . Solid black symbols correspond to experimental results of ref. ${ }^{19}$ Dotted lines indicate the expected scalings for $\mathcal{M} \gg 1$. evaporation) eventually leads to complete drying of the film.

Two asymptotic regimes exist for the deposit thickness and evaporation length considering $\mathcal{M} \gg 1$ and $\mathcal{M} \ll 1$. They are clearly demonstrated in the master curves displayed in Figure 10.

- Case $\mathcal{M} \ll 1$. Under this assumption, eqs 53 and 56 yield

$$
\tilde{h}_{d} \simeq \mathcal{N} \quad \text { and } \quad \tilde{L}_{e v} \simeq \sqrt{2} .
$$

This is the standard case in which the evaporation length reduces to the meniscus length, $\tilde{L}_{m}$. In dimensional variables, we recover

$$
h_{d} \simeq L_{m} \frac{\varphi_{0}}{\varphi_{c}-\varphi_{0}} \frac{v_{e v}}{V} .
$$

- Case $1 \ll \mathcal{M}$. In this case, we obtain

$$
\tilde{h}_{d} \simeq 2 \mathcal{M N} \text { and } \quad \tilde{L}_{e v} \simeq \sqrt{8} \mathcal{M},
$$

which corresponds to $L_{e v} \simeq L_{c}$ in dimensional variables. This asymptotic regime corresponds to the model developed by Jung and Ahn. ${ }^{19}$ The dimensional thickness is

$$
h_{d} \simeq a \frac{\varphi_{0}^{2}}{\left(\varphi_{c}-\varphi_{0}\right)^{2}} \frac{v_{e v}}{V^{2}} \frac{4 \kappa \gamma\left(1-\varphi_{c}\right)^{6}}{9 \eta_{0} \varphi_{c}} .
$$

For more insight into these limiting regimes, one can also show, using eq 50 and the above definition of $\mathcal{M}$, that

$\mathcal{M}=\frac{L_{c}^{2}}{2 L_{m}\left(L_{c}+L_{m}\right)}=\frac{\kappa \gamma}{a} \frac{h_{d}}{v_{e v} L_{e v}} \frac{k\left(\varphi_{c}\right)}{\eta_{0}} \frac{1}{L_{m}} .(61)$

The two asymptotic regimes thus correspond to $L_{c} \ll L_{m}$ and $L_{c} \gg L_{m}$, respectively. More interestingly, the above equation also suggests that $\mathcal{M}$ compares two different pressures: the critical capillary pressure $P_{\text {cap }}=\kappa \gamma / a$ and the pressure $P_{c}=\left(\eta_{0} / k\left(\varphi_{c}\right)\right)\left(L_{e v} / h_{d}\right) v_{e v} L_{m}$. This last pressure may be understood as follows: $\left(L_{e v} / h_{d}\right) v_{e v}$ is the scale of the velocity of the evaporation-induced solvent flow within the solvent-saturated film (relative to that of the colloids convected by the substrate), and 
$\nabla P=\left(\eta_{0} / k\left(\varphi_{c}\right)\right)\left(L_{e v} / h_{d}\right) v_{e v}$ is therefore the scale of the expected liquid pressure gradient. The unitless number $\mathcal{M}$ thus compares $P_{\text {cap }}$ to the typical (pervadic) pressure estimated using the pressure gradient caused by the relative flow within the film, on the scale of the meniscus $L_{m}$. The regime $\mathcal{M} \gg 1$ thus corresponds to a critical pressure larger than this pressure, i.e., $P_{\text {cap }} \gg \nabla P L_{m}$. Conversely, one encounters the second regime when the critical capillary pressure is smaller than the pressure that would cause the relative flow of solvent/colloids in the convected film.

\section{Comparison of the simplified model with experiments}

The experimental results of Jung and Ahn were reported (Figure 10, solid circles), assuming total wetting and using the properties of ethanol. As their experimental results concern the length of the wet dense-packed film, they correspond to $L_{c}=L_{e v}-L_{m}$ in our notation. To obtain the total length $L_{e v}$, the meniscus height corresponding to the dip coating configuration of their experiments (i.e., $\sqrt{2} \ell_{c}$, where $\ell_{c}$ is the capillary length) was added to their data. $\ell_{c}$ is used as the length scale so that the dimensionless meniscus height is $\sqrt{2}$, as in the present work. As shown in Figure 10, these data show very good agreement with the master curve. They are located in the transition region, which explains why the comparison with the asymptotic model developed by Jung and Ahn, which corresponds to $\mathcal{M} \gg 1$, was not accurate.

Note that two behaviors were observed in the experiments performed on colloidal dispersions in the evaporative regime performed by our group. Some of the experiments produced flat dry films. The dry film thicknesses obey the proportionality relation of eq 50 with $\tilde{L}_{e v} \simeq \sqrt{2}$. The corresponding process parameters ${ }^{13}$ yield a value of $\mathcal{M}$ in the range $0.005 \lesssim \mathcal{M} \lesssim 1$, which is consistent with the observed thicknesses. In other experiments, ${ }^{45}$ no steady state was obtained, and the dry films showed regular undulations (periodic regime with self-patterning). These last experiments correspond to larger values of the parameter $\mathcal{M}: 0.7 \lesssim \mathcal{M} \lesssim 10$. These results support the possibility of an instability mechanism in the regime of large $\mathcal{M}$. This possibility will be explored in a future work.

\section{Conclusion}

This work focuses on the drying of a colloidal dispersion in a flow coating process. We considered the evaporative regime encountered at low substrate velocity, where evaporation starts in the meniscus and continues in the wet densepacked film specific to colloidal dispersions. We developed an original model based on the general transport equations for binary mixtures, which can describe continuously the formation of the dry coating starting from the dilute colloidal dispersion. This model uses a no-slip boundary condition at the substrate for the solute velocity, which makes it possible to obtain a flat dense-packed wet film, as expected. The transition from the wet to the dry film is obtained through a pressure condition related to pore opening. Analysis of numerical simulations shows the existence of two regimes depending on the process parameters (substrate velocity, evaporation velocity, bulk concentration, and particle size). We also derived a simple analytical prediction for the dry film thickness $h_{d}$, which is in very good agreement with our numerical simulations. For some range of the process parameters, the total evaporation length is close to the meniscus size, and the model recovers the proportionality relation $h_{d} \propto \varphi_{0} v_{e v} / V$ observed in our experiments. However, the model shows the existence of another regime that is characterized by an evaporation length much larger than the meniscus and leads to another proportionality relation for the dry film thickness $\left(h_{d} \propto \varphi_{0}^{2} v_{e v} / V^{2}\right)$. This regime corresponds to the solution first presented by Jung and Ahn. ${ }^{19}$ Therefore, the theory presented in this work unifies the different approaches in the literature and is consistent with previous experimental results. ${ }^{13,19}$

The above results were obtained in a steadystate situation, and it will be interesting in a future work to explore the transient regime. Indeed, experimental self-patterning ${ }^{45}$ was ob- 
served for large values of $\mathcal{M}$, which motivates exploration of the possibility of an instability mechanism in this regime.

Acknowledgement The authors thank Daniel Lhuillier (retired from IJLRA-CNRS) for useful discussion and ANR EVAPEC (13BS09-0010-01) for funding.

\section{References}

(1) Sondergaard, R.; Hösel, M.; Angmo, D.; Larsen-Olsen, T.; Krebs, F. C. Roll-to-roll fabrication of polymer solar cells. Mater. Today 2012, 15, 36-49.

(2) Mirri, F.; Ma, A.; Hsu, T.; Behabtu, N.; Eichmann, S.; Young, C.; Tsentalovich, D.; Pasquali, M. HighPerformance Carbon Nanotube Transparent Conductive Films by Scalable Dip Coating. ACS Nano 2012, 6, 9737-9744.

(3) Malaquin, L.; Kraus, T.; Schmid, H.; Delamarche, E.; ; Wolf, H. Controlled Particle Placement through Convective and Capillary Assembly. Langmuir 2007, 23, 11513-11521.

(4) Prevo, B. G.; Kuncicky, D. M.; Velev, O. D. Engineered deposition of coatings from nano- and micro-particles: A brief review of convective assembly at high volume fraction. Colloids Surf., A 2007, 311, 2-10.

(5) Born, P.; Munoz, A.; Cavelius, C.; Kraus, T. Crystallization Mechanisms in Convective Particle Assembly. Langmuir 2012, 28, 8300-8308.

(6) Yabu, H.; Shimomura, M. Preparation of Self-Organized Mesoscale Polymer Patterns on a Solid Substrate: Continuous Pattern Formation from a Receding Meniscus. Adv. Funct. Mater. 2005, 15, 575-581.

(7) Han, W.; Lin, Z. Learning from "Coffee Rings" Ordered Structures Enabled by Controlled Evaporative Self-Assembly.
Angew. Chem., Int. Ed. 2012, 51, 15341546.

(8) Brewer, D. D.; Shibuta, T.; Francis, L.; Kumar, S.; Tsapatsis, M. Coating Process Regimes in Particulate Film Production by Forced-Convection-Assisted Drag-Out. Langmuir 2011, 27, 11660-11670.

(9) Davis, R.; Jayaraman, S.; Chaikin, P.; Register, R. Creating Controlled Thickness Gradients in Polymer Thin Films via Flowcoating. Langmuir 2014, 30, 56375644 .

(10) Dimitrov, A. S.; Nagayama, K. Continuous Convective Assembling of Fine Particles into Two-Dimensional Arrays on Solid Surfaces. Langmuir 1996, 12, 1303-1311.

(11) Berteloot, G.; Daerr, A.; Lequeux, F.; Limat, L. Dip coating with colloids and evaporation. Chem. Eng. Proc. 2013, 68, 69-73.

(12) Le Berre, M.; Chen, Y.; Baigl, D. From convective assembly to Landau-Levich deposition of multilayered phospholipid films of controlled thickness. Langmuir 2009, 25, 2554-2557.

(13) Jing, G.; Bodiguel, H.; Doumenc, F.; Sultan, E.; Guerrier, B. Drying of Colloidal Suspensions and Polymer Solutions near the Contact Line: Deposit Thickness at Low Capillary Number. Langmuir 2010, 26, 2288-2293.

(14) Grosso, D. How to exploit the full potential of the dip-coating process to better control film formation. J. Mater. Chem. 2011, 21, 17033-17038.

(15) Routh, A. F.; Russel, W. B. Horizontal Drying Evaporation Fronts During Solvent from Latex Films. AIChE J. 1998, 44, 2088-2098.

(16) Goehring, L.; Clegg, W. J.; Routh, A. F. Solidification and ordering during directional drying of a colloidal dispersion. Langmuir 2010, 26, 9269-9275. 
(17) Routh, A. F. Drying of thin colloidal films. Rep. Prog. Phys. 2013, 76, 046603.

(18) Doumenc, F.; Guerrier, B. Drying of a Solution in a Meniscus: A Model Coupling the Liquid and the Gas Phases. Langmuir 2010, 26, 13959-13967.

(19) Jung, Y. D.; Ahn, K. H. Prediction of Coating Thickness in the Convective Assembly Process. Langmuir 2013, 29, 15762-15769.

(20) Peppin, S. S.; Elliott, J. A.; Worster, M. G. Solidification of colloidal suspensions. J. Fluid Mech. 2006, 554, 147-166.

(21) Style, R. W.; Peppin, S. S. Crust formation in drying colloidal suspensions. Proc. R. Soc. A 2011, 467, 174-193.

(22) Parneix, C.; Vandoolaeghe, P.; Nikolayev, V. S.; Quéré, D.; Li, J.; Cabane, B. Dips and Rims in Dried Colloidal Films. Phys. Rev. Lett. 2010, 105, 266103.

(23) Wedin, P.; Martinez, C. J.; Lewis, J. A.; Daicic, J.; Bergström, L. Stress development during drying of calcium carbonate suspensions containing carboxymethylcellulose and latex particles. J. Colloid Interface Sci. 2004, 272, 1-9.

(24) Lidon, P.; Salmon, J. Dynamics of unidirectional drying of colloidal dispersions. Soft Matter 2014, 10, 4151.

(25) Deegan, R. D.; Bakajin, O.; Dupont, T. F.; Huber, G.; Nagel, S. R.; Witten, T. Contact line deposits in an evaporating drop. Phys. Rev. E 2000, 62, 756.

(26) Bird, B. R.; Stewart, E. W.; Lightfoot, E. N. Transport phenomena; Wiley international edition, 2002.

(27) Vollebregt, H. M.; van der Sman, R. G. M.; Boom, R. M. Suspension flow modelling in particle migration and microfiltration. Soft Matter 2010, 6, 6052 - 6064 .
(28) Nägele, G. The Physics of Colloidal Soft Matter; Polish Academy of Sciences publication: Warsaw, 2004.

(29) Russel, W. B.; Saville, D. A.; Schowalter, W. R. Colloidal dispersions; Cambridge University Press, 1989.

(30) Pusey, P. N.; van Megen, W. Phase behavior of concentrated suspensions of nearly hard colloidal spheres. Nature 1986, 320, 340-342.

(31) Brown, L. A.; Zukoski, C. F.; White, L. R. Consolidation During Drying of Aggregated Suspensions. AIChE J. 2002, 48, 492-502.

(32) Daubersies, L.; Salmon, J.-B. Evaporation of solutions and colloidal dispersions in confined droplets. Phys. Rev. E 2011, 84, 031406 .

(33) Merlin, A.; Salmon, J.-B.; Leng, J. Microfluidic-assisted growth of colloidal crystals. Soft Matter 2012, 8, 3526-3537.

(34) Gromer, A.; Nassar, M.; Thalmann, F.; Hébraud, P.; Holl, Y. Simulation of Latex Film Formation Using a Cell Model in Real Space: Vertical Drying. Langmuir 2015, 31, 10983-10994.

(35) Kaplan, C.; Mahadevan, L. Evaporationdriven ring and film deposition from colloidal droplets. J. Fluid Mech. 2015, 781.

(36) Kaplan, C.; Wu, N.; Mandre, S.; Aizenberg, J.; Mahadevan, L. Dynamics of evaporative colloidal patterning. Phys. Fluids 2015, 27, 092105.

(37) Peppin, S. S.; Elliott, J. A.; Worster, M. G. Pressure and relative motion in colloidal suspensions. Phys. Fluids 2005, 17, 053301.

(38) Nozieres, P. A local coupling between sedimentation and convection: application to the Beenaker-Mazur effect. Phys. A 1987, 219-237. 
(39) Lhuillier, D. Internal variables and the non-equilibrium thermodynamics of colloidal suspensions. J. Non-Newtonian Fluid Mech. 2001, 96, 19-30.

(40) Larson, R. G. The Structure and Rheology of Complex Fluids; Oxford University Press: New York, 1999.

(41) Oron, A.; Davis, S.; Bankoff, S. Long-scale evolution of thin liquid films. Rev. Mod. Phys. 1997, 69, 931.

(42) Lyushnin, A. V.; Golovin, A. A.; Pismen, L. M. Fingering instability of thin evaporating liquid films. Phys. Rev. E 2002, 65, 021602.

(43) Frastia, L.; Archer, A. J.; Thiele, U. Modelling the formation of structured deposits at receding contact lines of evaporating solutions and suspensions. Soft Matter 2012, 8, 11363.

(44) Riddick, J. A.; Bunger, W. B.; Sakano, T. Organic solvents, Physical Properties and Methods of Purification, Vol. II; John Wiley: New York, 1986.

(45) Bodiguel, H.; Doumenc, F.; Guerrier, B. Stick- slip patterning at low capillary numbers for an evaporating colloidal suspension. Langmuir 2010, 26, 1075810763. 


\section{Graphical TOC Entry}

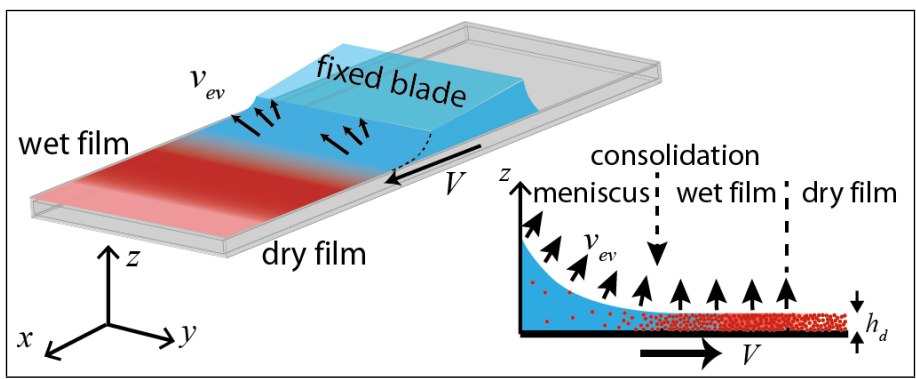

